\title{
СПЕКТРЫ ОТРАЖЕНИЯ И ПОГЛОЩЕНИЯ МОНОКРИСТАЛЛОВ CaS ВБЛИЗИ КРАЯ ФУНДАМЕНТАЛЬНОГО ПОГЛОЩЕНИЯ
}

Измерены спектры отражения монокристаллов $\mathrm{CaS}$ в интервале $2-6,3$ эB. Первый максимум в спектре находится при 5,3 эB. В спектрах пропускания сильный рост поглощения $x>30 \mathrm{~cm}^{-1}$ обнаруживается при 4,4 эВ. Обсуждается возможность привлечения непрямых $\Gamma-X$ переходов, которые по существующим расчетам зонной стружтуры $\mathrm{A}^{\mathrm{II}} \mathrm{B}^{\mathrm{VI}}$ могут оказаться самыми низкоэнерпетичеокими межзонными переходами, для объяснения оптических явлений, наблюдаемых на краю фундаментального поглощения $\mathrm{CaS}$.

Данные об электронной структуре сульфидов щелочноземельных металлов получены путем измерения поглощения пленок, изготовленных обработкой тонкого слоя металла в сере $\left[{ }^{1}\right]$, либо катодным напылением и термической сублимацией $\left[{ }^{2,3}\right]$. Измерялось также отражение прессованных порошков и пленок, полученных катодным напылением $\left[{ }^{4}\right]$. Из-за структурного несовершенства таких объектов получаются не совсем однозначные данные. Так, например, из данных отражения пленок $\left[{ }^{2}\right]$ край фундаментального поглощения $\mathrm{CaS}$ оценен $5,1 \pm 0,3$ эB. Такой разброс создает весьма большую неопределенность при интерпретации зонной структуры $\mathrm{CaS}$, спектров возбуждения люминесценции и трактовке электронно-дырочных и экситонных процессов в нем.

Поскольку $\mathrm{CaS}$, типичный представитель соединений $\mathrm{A}^{\amalg} \mathrm{B}^{\mathrm{vI}}$, является основой и ряда разработок прикладного характера, вопросы о его зонной структуре, о природе спектров фундаментального поглощения, о начале межзонных переходов становятся особенно актуальными.

\section{Методика эксперимента}

Монокристаллы выращивались нами по методике, описанной в $\left[{ }^{5}\right]$. Образцы получились весьма небольших линейных размеров - в среднем около 0,5 мм, в последнее время удалось увеличить их размеры до $2-3 \times 3$ мм. По данным химического и рентгеноструктурного анализов синтезированные монокристаллы были идентифицированы как сульфид кальция.

Спектры поглощения монокристаллов $\mathrm{CaS}$ измерялись на спектрофотометре СФ-16. Коэффициент отражения свежеизготовленных или свежесколотых монокристаллов $\mathrm{CaS}$ определялся с помощью монохроматора спектрофотометра СФ-16. Источником света служила дейтериевая лампа ДДС-30, приемником - ФЭУ-39. Измерения проводились при комнатной температуре, угол падения и отражения $45^{\circ}$. (Обработка таких спектров описана в [ $\left.{ }^{6}\right]$.) 
Измерение отражения от монокристаллов малых размеров связано с рядом трудностей, основной из которых является та, что при падении светового пучка освещается не только нужная грань кристаллика, но и другие его грани и детали укрепления (обычно графитовая подложка, которая в исследуемой нами спектральной области до 6,3 э $B$ имеет слабое неселективное рассеяние). Мы учли вклад такого света вычитанием из общего сигнала той его доли, которая сохраняется в условиях, когда зеркально отраженный луч (блик) не попадает на фотокатод ФЭУ.

Пока ограничен спектральный интервал - невозможно решить проблему спектральной трансформации отраженного луча малой интенсивности (такая трансформация необходима в вакуумной ультрафиолетовой области).

Для измерений нами были использованы лишь свежевыращенные объекты, поскольку получить свежую поверхность кристаллика путем окалывания удается редко. При хранении кристаллик начинает давать желтоватые блики (при дневном свете), свидетельствующие о росте рассеяния начиная с синей области спектра. Из-за такого рассеяния спектр отражения искажается, и хотя на фоне рассеяния все же проявляются слабые пики, соответствующие полосам фундаментального поглощения, количественно обработать эти спектры невозможно. Отражение от пленок и прессованных образцов сопровождается, как правило, из-за рассеяния аналогичными искажениями.

\section{Спектры отражения и поглощения}

На рис. 1 показан спектр отражения выращенных нами монокристаллов непосредственно после изготовления. Этот спектр хорошо повторяет

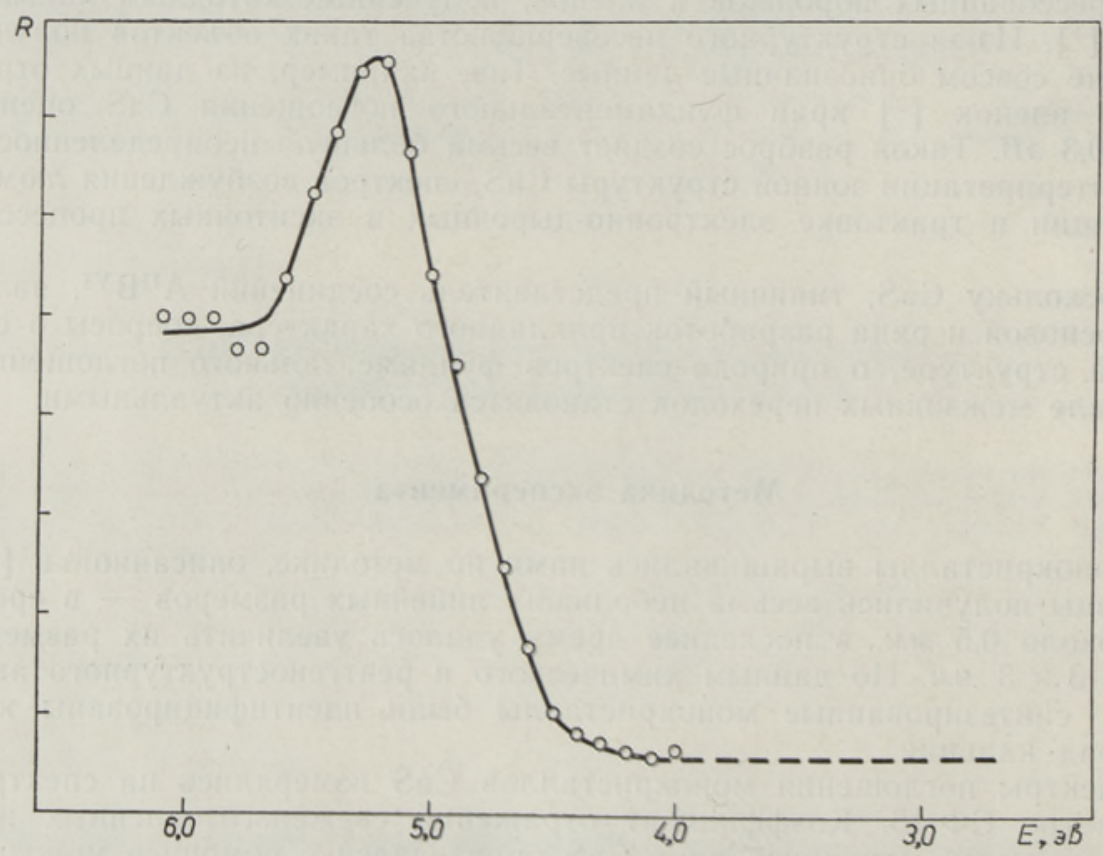

Рис. 1. Спектр отражения свежевыращенного монокристалла CaS.

спектр поглощения Саума и Хенсли [ $\left.{ }^{1}\right]$ с максимумом при 5,3 э $B$; никаких особенностей в интервале $4,5-5,0$ э $B$ не обнаружено. 
Анализ этого спектра путем применения преобразования КрамерсаКронига из-за ограниченного спектрального интервала невозможен. Все же из спектрального хода отражения непосредственно следует, что самостоятельных прямых зонных піереходов в области $E<5,0$ э $B$ не наблюдается. Это заключение находится в хорошем согласии с результатом, полученным нами еще в 1969 г. при изучении спектров возбуждения рекомбинационной люминесценции $\mathrm{CaS}-\mathrm{Bi}$-фосфоров $\left[{ }^{7}\right]$. Тогда было установлено, что переход от коэффициентов поглощения, характерных для фундаментального поглощения $\left(10^{5}-10^{4} \mathrm{~cm}^{-1}\right)$, к коэффициентам, характерным для примесного поглощения или для непрямых межзонных переходов $\left(\varkappa \leqslant 10^{2} c M^{-1}\right)$, происходит в спектральном интервале $5,0-4,9$ эB. В таком случае полосы поглощения и возбуждения в области $4,5-4,9$ эВ соответствуют вероятнее всего либо околопримесному поглощению, либо непрямым переходам, и лишь полосы в области $E>4,9$ э $B$ - возбуждению на краю фундаментального поглощения, связанному с прямыми переходами.

Поскольку метод отражения в области малых коэффициентов поглощения практически не применим, выяснение природы поглощения в области $E \leqslant 4,9$ эB требует привлечения других методик. Предпочтительнее всего здесь измерение пропускания, спектральная зависимость которого позволяет определить, обусловлен ли край собственного поглощения прямыми или непрямыми переходами. Как известно (см., напр., $\left.\left[{ }^{8}\right]\right)$, спектральная зависимость коэффициента поглощения $x$ в случае непрямых переходов имеет вид

$$
x=B / h v\left(h v-E_{g}\right)^{2} \text {, }
$$

а в случае прямых переходов - вид

$$
x=A / h v\left(h v-E_{g}\right)^{1 / 2} .
$$

В качестве первого шага в этом направлении мы измерили пропускание безйодных монокристаллов $\mathrm{CaS}$ при комнатной температуре (рис. 2) и нашли сильный рост поглощения свыше 30 $c \mathcal{M}^{-1}$ при 4,4 эB, при бо́льших энергиях кванта поглощение нигде не опускается ниже $\sim 10^{2} \mathrm{CM}^{-1}$. Экстраполяция данных рис. 2 по формуле (1) к энергиям фотона $E=$ $=4,8{ }{ }^{B}$ дает $\varkappa_{4,8}=$ $=130 \mathrm{~cm}^{-1}$, что нахо-

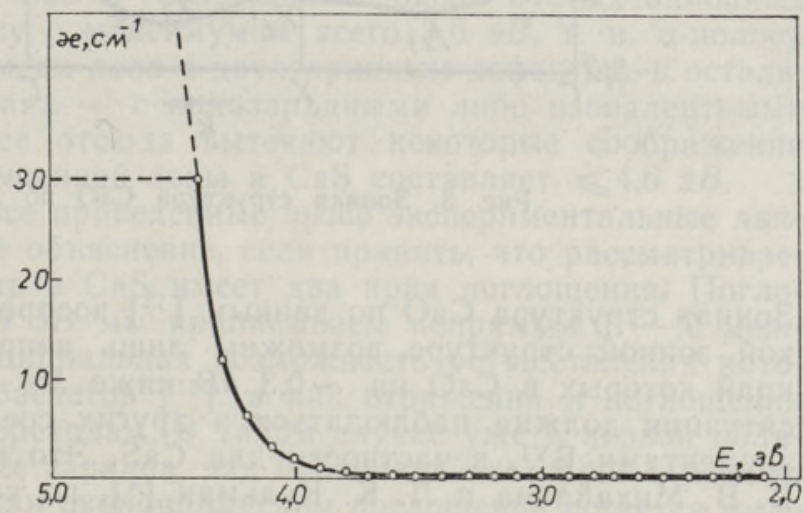

Рис. 2. Спектр поглощения монокристалла $\mathrm{CaS}$ при комнатной температуре.

дится в хорошем согласии с результатами [ $\left.{ }^{9}\right]$ (см. также ниже). Неплохо согласуются с формулой (1) и данные, полученные обработкой спектров диффузного отражения чистого $\mathrm{CaS}$ из работы $\left[{ }^{10}\right]$, тогда как согласие с формулой (2) отсутствует. Мы пришли к заключению, что поглощение в рассматриваемой области не может быть обусловлено прямыми зонными переходами. Остается выяснить, связано ли оно с непрямыми межзонными переходами или с переходами около дефектов решетки. 
O зонной структуре соединений $\mathrm{A}^{\mathrm{II}} \mathrm{B}^{\mathrm{VI}}$ и особенностях длинноволновой части фундаментального поглощения $\mathrm{CaS}$

При переходе от соединений магния к соединениям кальция зонная структура в этом классе веществ существенно меняется. Как известно, в MgO первая полоса фундаментального поглощения связана с экситонным дублетом в Г-точке зоны Бриллюэна $\left[{ }^{11}\right]$. В $\mathrm{CaO}$, по расчетам зонной структуры $\left[{ }^{12,13}\right]$, самыми низкоэнергетическими являются переходы в точке $X$ из-за сильного понижения состояния $X_{3}$, вызванного наличием пустой $d$-орбитали в атомах Са (полоса поглощения при 6,8 э $B$ в $\mathrm{CaO}$ ). В этом случае максимум валентной зоны сохраняется в точке $\Gamma$.

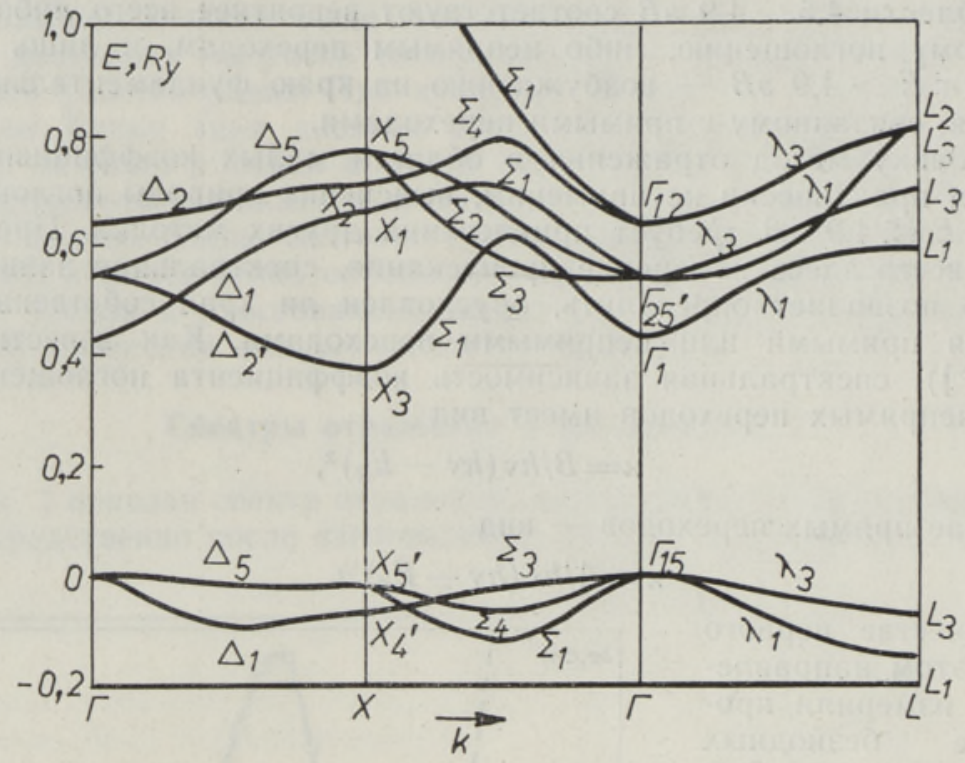

Рис. 3. Зонная структура $\mathrm{CaO}$ по данным $\left[{ }^{13}\right]$.

Зонная структура $\mathrm{CaO}$ по данным $\left[{ }^{13}\right]$ воспроизведена на рис. 3 . В такой зонной структуре возможны лишь непрямые $\Gamma_{15}-X_{3}$ переходы, край которых в СаO на $\sim 0,3{ }{ }^{B}$ ниже $X_{5}^{\prime}-X_{3}$ края $\left[{ }^{13}\right]$. Такая же ситуация должна наблюдаться в других соединениях $\mathrm{Ca}, \mathrm{Sr}$ и $\mathrm{Ba}$ с элементами B $\mathrm{BI}^{\mathrm{I}}$, в частности для CaS. Это подтверждается оценками В. В. Михайлина и Л. К. Кульман $\left[{ }^{4}\right]$, по которым зонная структура $\mathrm{CaS}$ подобна зонной структуре $\mathrm{CaO}$.

В работе $\left[{ }^{11}\right]$ предполагалось интерпретировать первую полосу поглощения $\mathrm{CaO}$ усложненным Г-экситоном (см. также $\left.\left[{ }^{14,15}\right]\right)$, однако подробный анализ в $\left[{ }^{12}\right]$ эту возможность опровергает.

При интерпретации первой полосы поглощения $\mathrm{CaO}$ при 6,8 эB как переходов $X_{5}^{\prime}-X_{3}$ возможны два случая: 1) возникновение экситонных состояний, 2) межзонные переходы. Выбор между этими двумя возможностями пока не осуществлен.

Проанализируем экспериментальные данные о зонной структуре $\mathrm{CaS}$.

1. В работе $\left[{ }^{1}\right]$ первому максимуму фундаментального поглощения $\mathrm{CaS}$ при 5,3 э $B$ приписывалась экситонная природа. В более тяжелых соединениях $\mathrm{A}^{\text {IB }}{ }^{\mathrm{VI}}$ ясно обнаруживается дублетная структура экситонной полосы, которая обусловлена спин-орбитальным взаимодей- 
ствием в ионах. С учетом вероятной энергии связи этого экситона 0,30,5 э $B$ ширина запрещенной зоны $\mathrm{CaS}$ в соответствующей точке зоны Бриллюэна ожидается в интервале 5,6-5,8 эB $\left[{ }^{2}\right]$.

2. Из данных $\left.{ }^{16}\right]$ по фотонному умножению в $\mathrm{CaS}$ (начиная с 11 э $B$ ) следует, что ширина запрещенной зоны не превышает $\sim 5,5$ эB (в случае узкой валентной зоны), но может быть и значительно у́же (вплоть до значения $11 / 4$ эB), если валентная зона широкая и эффективные массы электрона и дырки примерно равные. Отметим, что валентная зона $\mathrm{CaS}$ скорее всего не узкая (порядка $\sim 4{ }_{\text {э }} B$ ). М. Кинк наблюдала рост выхода люминесценции $\mathrm{CaS}-\mathrm{Bi}$ при $E_{\text {возб. }}=8,5$ э $B$, что в случае ударного механизма возбуждения ионов $\mathrm{Bi}^{3+}$ (первая полоса возбуждения $\mathrm{Bi}^{3+}$ в $\mathrm{CaS}-3,0$ эB) также ведет к значению $E<5,5$ эВ $\left[{ }^{17}\right]$.

3. В неактивированном $\mathrm{CaS}$ ощутимый рост поглощения (по диффузному отражению $\left[{ }^{10}\right]$ в порошках и по прямым определениям поглощения на монокристаллах $\left[{ }^{5}\right]$, содержащих, однако, много йода) наблюдается уже с 4,4-4,5 әB. По оценкам В. Лемана [ $\left.{ }^{9}\right]$, рост коэффициента поглощения в его образцах (из данных по диффузному отражению) выше значения $10^{2}$ происходит при 4,8 эB.

4. В спектрах возбуждения люминесценции $\mathrm{CaS}$ наблюдаются полосы 4,6-4,9 эB, в этой же области обычно эффективно возбуждается и рекомбинационная люминесценция. Особенно обращает на себя внимание в этом отношении спектр возбуждения рекомбинационной люминесценции $\mathrm{CaS}-\mathrm{Mn}$ в $\left[{ }^{17}\right]$ с максимумом при 4,9 э $B$ и простирающийся до 4,4 эB, что свидетельствует о процессах разделения зарядов в $\mathrm{CaS}$ в этой спектральной области. Ранее мы приписывали эти полосы околоактиваторным возбуждениям $\left[{ }^{18}\right]$, но эта интерпретация не была окончательной. Единственный надежно идентифицированный тип околодефектного возбуждения в $\mathrm{CaS}$ - возбуждение около пустых анионных вакансий - имеет полосу с максимумом всего 3,6 э $B$, т. н. $\alpha$-полосу $\left[{ }^{19}\right]$. Правда, здесь мы имеем дело с двухзарядным дефектом, в остальных рассмотренных случаях - с однозарядными либо изовалентными дефектами. Тем не менее отсюда вытекают некоторые соображения о том, что ширина запрещенной зоны в $\mathrm{CaS}$ составляет $\leqslant 4,6$ э $B$.

По нашему мнению, все приведенные выше экспериментальные данные находят естественное объяснение, если принять, что рассматриваемая спектральная область в $\mathrm{CaS}$ имеет два края поглощения. Поглощение в области $E<4,9$ э $B$ мы приписываем непрямым $\Gamma-X$ межзонным переходам, принципиальная возможность осуществления которых следует из зонных расчетов $\left[{ }^{13}\right]$, а пик отражения и поглощения при 5,3 э $B$ - прямым переходам. В таком случае уже в первой области происходит разделение зарядов, ряд проявлений которого в рамках концепции о примесном или околопримесном поглощении остается мало понятным. Надо, однако, учесть, что на относительно слабое поглощение, обусловленное непрямыми переходами, может налагаться и давать ощутимый вклад также примесное поглощение, так что обе затронутые концепции являются отнюдь не взаимоисключающими.

Авторы благодарны В. Г. Плеханову за полезные обсуждения, А. Кильку за изготовление безйодных монокристаллов, А. Хааву за проведение рентгеноструктурного анализа и А. С. Кузнецову за помощь в экспериментах.

\section{Л И ТЕ Р А Т У Р А}

1. S a um, Y., Hensley, E. B., Phys. Rev., 113, 1019 (1959).

2. Levshin, V. L., Mikhailin, V. V., Proc. Intern. Conf. on Luminescence, 1966, pp. $1508-1513$. 
3. Левшин В. Л., Михайлин В. В., Саулевич Л. К., Изв. АН СССР, сер. физ., 33, 974 (1969).

4. Кульман Л. К., Михайлин В. В., Тр. ИФА АН ЭССР, № 41, 3 (1972).

5. Р е а ло К. В., Тр. ИФ АН ЭССР, № 43, 260 (1975).

6. Ro e s s le r, D. M., Brit. J. Appl. Pliys., 16, 1359 (1965).

7. Егоров а Л. С., Окк (Кинк) М. Ф., Я эк И. В., Тр. ИФА АН ЭССР, № 35, 76 (1969).

8. Johnson, E. J., В кн.: Оптические свойства полупроводников, М., 1970, c. $201-205$.

9. Le h m a n, W., J. Electrochem. Soc., 117, 1389 (1970).

10. Л ущик Н. Е., Мерилоо И. А., Тр. ИФА АН ЭССР, № 35, 56 (1969).

11. Roessler, D. M., W a lker, W. C., Phys. Rev., 159, 733 (1967).

12. Whited, R. C., W alker, W. C., Phys. Rev., 188, 1380 (1969).

13. S eth, U., Chan ey, R., Phys. Rev., B, 12, 5923 (1975).

14. D a ud e, N., J o u a ni in, C., Gout, C., Phys. stat. sol. (b), 72, K173 (1975).

15. Улььици Б., У льрици В., Ковалев Н. Н., ФТТ, 17, 1533 (1975).

16. Лущик Н. Е., Лущик Ч. Б., МерилооИ. А., Соовик Х. А., Тр. ИФА АН ЭССР, № 34, 49 (1966).

17. Кин к М. Ф., Тр. ИФ АН ЭССР, № 43, 179 (1975).

18. Я્Яэ К И. В., Х ю т Т Г. И., Изв. АН СССР, сер. физ., 35, 1505 (1971).

19. К уз нецов А. С., Тр. ИФ АН ЭССР, № 43, 192 (1975).

$\begin{array}{cc}\text { Ннститут физики } & \text { Поступила в редакцию } \\ \text { Академии наук Эстонской ССР } & 11 / \mathrm{XI} 1976\end{array}$

K. REALO, I. JAEK

\section{CaS MONOKRISTALLIDE PEEGELDUMIS- JA NEELDUMISSPEKTRID POHINEELDUMISE ÄAREL}

Mõõdeti CaS monokristallide peegeldumis- ja neeldumisspektrid energiavahemikus $2 \div 6,3 \mathrm{eV}$. Peegeldumisspektri maksimum asub $5,3 \mathrm{eV}$ juures, neeldumisspektris täheldati neeldumise järsku suurenemist $4,4 \mathrm{eV}$ juures $\left(x>30 \mathrm{~cm}^{-1}\right)$. Pikalainelise riba tekkimist $\mathrm{CaS}$ pōhineeldumise äärel võib oletatavasti seostada kaudsete $\Gamma-X$-üleminekutega, mis $\mathrm{A}^{\mathrm{II}} \mathrm{B}^{\mathrm{VI}}$-tüüpi ühendite tsoonistruktuuri arvutuste põhjal võivad põhjustada kõige madalama energiaga tsoonidevahelisi üleminekuid.

K. REALO, I. JAEK

\section{REFLECTANCE AND ABSORPTION SPECTRA OF CaS SINGLE CRYSTALS AT THE EDGE OF FUNDAMENTAL ABSORPTION}

The reflectance spectra of $\mathrm{CaS}$ single crystals measured in the energy region from 2 to $6.3 \mathrm{eV}$ exhibited a peak at $5.3 \mathrm{eV}$. In the absorption spectra a strong increase up to $\varkappa>30 \mathrm{~cm}^{-1}$ at $4.4 \mathrm{eV}$ was observed. The possibility that indirect $\Gamma-X$-transitions may cause the most low-energetic peak at the edge of fundamental absorption of $\mathrm{CaS}$ is discussed. 